3

\title{
A Case Study on CAD/CAM data transfer in CIM Environment
}

\author{
K. P. Mahadevan \\ J. Mou \\ M. R. Henderson \\ D. L. Shunk \\ Department of I\&MSE \\ Arizona State University \\ Tempe, $A Z 85287$ \\ U.S.A.
}

\begin{abstract}
This case study enumerates the steps involved in testing the data exchange among dissimilar CAD systems using a standard exchange mechanism and discusses the level of inter-operability that can be achieved to facilitate successful transfer of CAD data among three CAD software's namely Pro/E version 17, I-DEAS Master series version 3.1 and AutoCAD release 13 in a CIM environment. First the Initial Graphics Exchange Specification (IGES) is used as the data exchange mechanism to test these software's using a standard test procedure. The same test procedure is then used to find out the effectiveness of the data exchange between Pro/E and IDEAS using a different exchange standard called the Standard For Exchange Of Product Model Data (STEP). AutoCAD does not support STEP. Experimental results show that Pro/E and I-DEAS have the capability to share complete CAD information but AutoCAD has some limitations in transferring information on surfaces and solids using IGES. By using the STEP translator, we are able to translate CAD data efficiently one way from Pro/E to I-DEAS and vice versa but have some difficulty carrying out back and forth data transfer between them.
\end{abstract}




\section{Keywords}

Computer Aided Design (CAD), Computer Aided Manufacturing (CAM), Computer Integrated Manufacturing (CIM), Inter-operability, Initial Graphics Exchange Specification (IGES), Standard For Exchange Of Product Model Data (STEP).

\section{INTRODUCTION}

Traditionally CAD systems have competed on ease and speed with which the geometry is created, but there is an increasing need for these systems to have the capability to integrate seamlessly with other systems in a CIM environment. The present day manufacturers are working concurrently with a wider range of dedicated suppliers, each having their own legacy systems with varying levels of technical sophistication. In addition to this these manufacturers and suppliers have access to more product information and a variety of CAD/CAM products to choose from. So it is very important for these suppliers to be able to effectively communicate vital product data with the manufacturers, quickly and effectively regardless of the system they have [1]. If this is achieved the manufacturers can obtain quick feedback from the suppliers on new designs, existing designs can be modified faster and product variations can be minimized by utilizing the existing standard components from the product database thereby reducing product development costs. To obtain these benefits of seamless integration we have to evaluate the effectiveness of the $\mathrm{CAD}$ data exchange among the systems available using a standard exchange mechanism. The effectiveness depends on the extent to which the data exchange satisfies the user's specific requirement working under a particular environment. Data is exchanged from the base system to the target system by first translating into a neutral file format and then to the data structure of the target system. Data exchange between any two dissimilar CAD systems can either be a simple data transfer involving simple geometry, or a complex model having a variety of entity types. So in order to get the desired results, it is important for one to understand the $\mathrm{CAD}$ application, how it is used, the operating system requirements, the hardware requirements, the mode of communication, knowledge of files and file transfer protocols.

The objective of this paper is to find out the compatibility of the three dissimilar CAD systems, namely AutoCAD 13 [2], I-DEAS 3.1 [3], and Pro/E 17 [4] using Initial Graphics Exchange Specification (IGES) as the standard for exchange of CAD data. IGES is the specification for the structure and syntax of the neutral file in binary or ASCII format. Here AutoCAD (supporting IGES 5.1) was operated in a Windows NT environment having a 64MB RAM with Pentium processor, IDEAS and Pro/E both supporting IGES 5.1, in a Silicon Graphics workstation with 64MB RAM, 2GB hard drive and MIPS R4600 (134 MHz) CPU. We see that the hardware requirements of Pro/E and I-DEAS are greater than AutoCAD. All the three softwares were on different servers. 


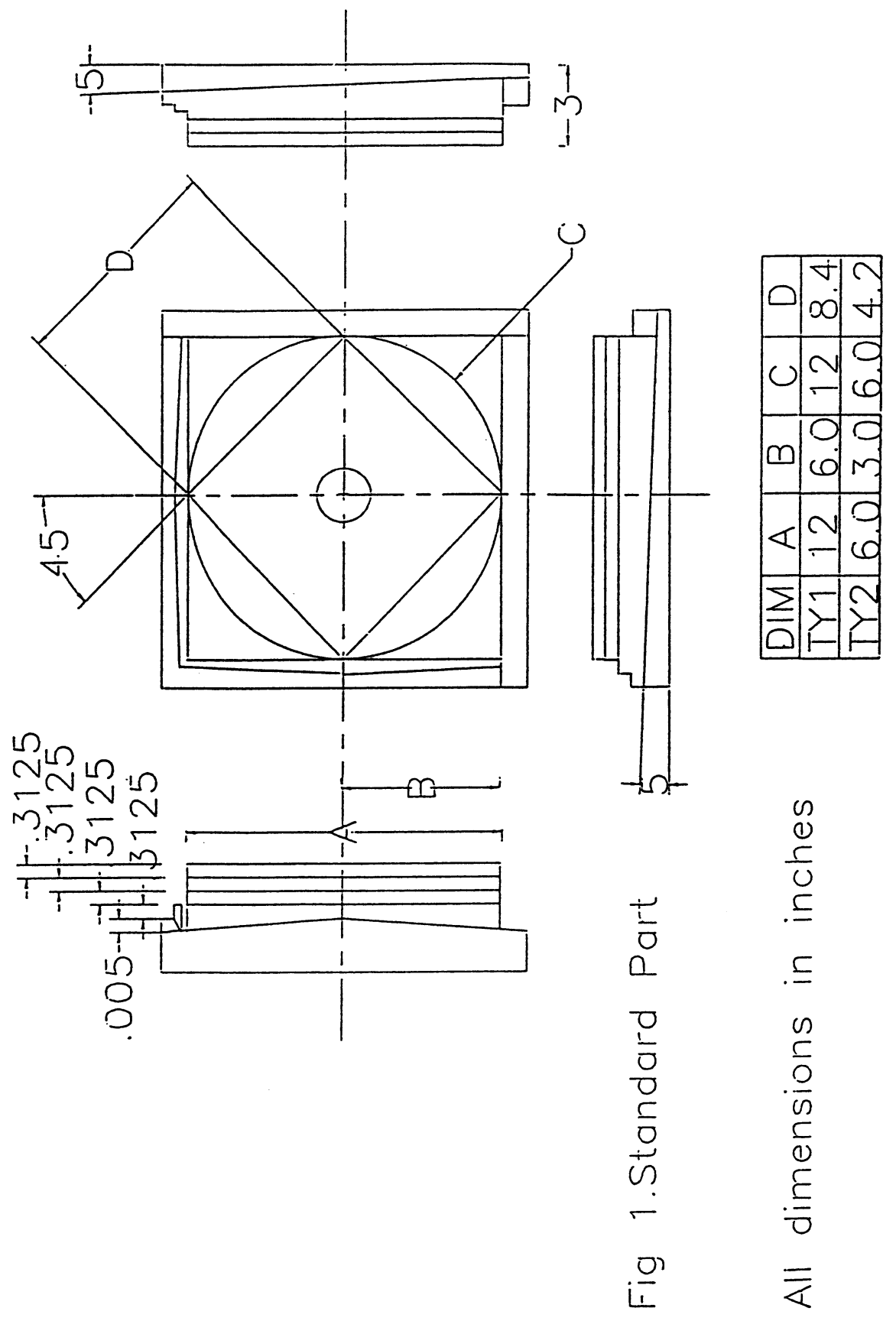




\section{TESTING PROCEDURE}

A standard drawing of the part, as shown in Figure 1, is created in each of the three softwares. This provides a common ground for comparison. The following steps have been carried out for each of the software to evaluate their compatibility.

\section{Step One}

Create a CAD file in the base system, generate the IGES file for the same using the export option (igesout). Check the .log file for any errors or warning message and identify the causes in the pre-processor. Then import it (igesin) into the same base system. Again check .log file for any error messages by the post-processor. Check whether the plots look the same and the geometry is correct. This method uses the pre-processor and post processor (translator) of the same CAD system. See Figure 2.

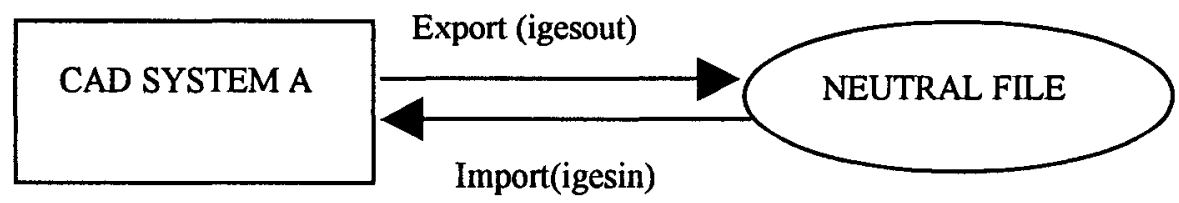

Figure 2 Step one of testing procedure

\section{Step Two}

Create a CAD file in system A, generate the IGES file and transfer this file to another CAD system say system B using File Transfer Protocol (FTP), see Figure 3. Before creating the IGES file in the base system A using the export option check the requirements of the receiving system $B$, like acceptable tolerances, type of representation of the model, so that the file created can be easily transferred to the receiving system. For example if the receiving system easily recognizes surfaces, then represent the solid model as a set of surfaces using the available IGES configurations or options in the base system.

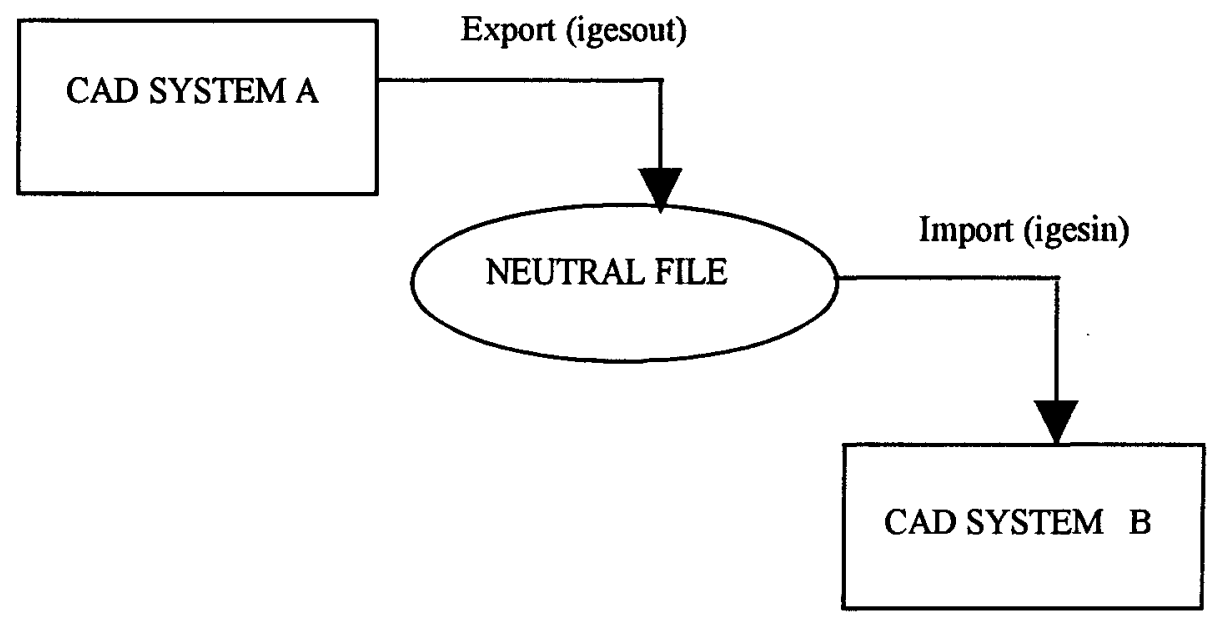


This facilitates easier transfer of data into the receiving system satisfying its requirements. Then recreate the solid in the receiving system by merging or stitching the set of surfaces into a solid. For the IGES file created in the base system, check the. $\log$ file for any errors and repeat the same for the receiving system. Check whether the plots look the same in both the systems. Look for any inconsistencies in the geometry and text. Also ensure that the units of the CAD file in both the systems are the same. One way to ensure that the units are the same is to check the mass properties like density, volume, surface area, etc. If the file is transferred completely without changing the form and function the mass properties will be the same. For example if the base system sends a solid, and the receiving system receives it as a wireframe or a set of surfaces, the resulting model will have no mass.

\section{Step Three}

Create a CAD file in system $A$, generate the IGES file and transfer this file to system B using FTP, create an IGES file of this transferred file in system B and transfer it back to system A using FTP. It should be noted that we do not recreate the model in system B. Thus this step involves repeating the Step Two twice, once from $A$ to $B$ and once from B back to A, see Figure 4 .

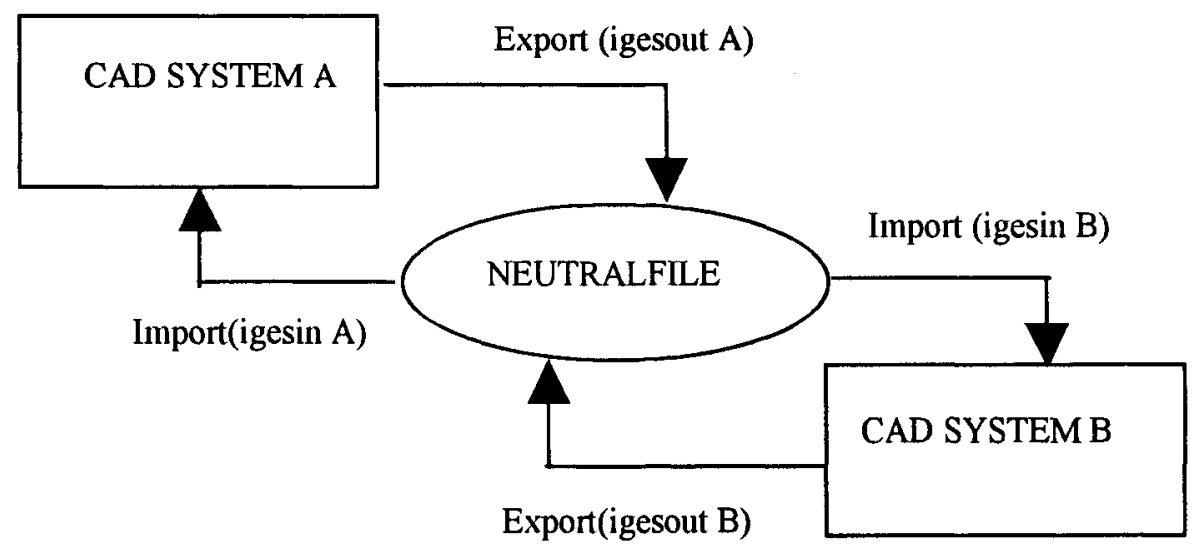

Figure 4 Step three of testing procedure

\section{OBSERVATIONS}

\section{(i) IGES}

\section{Pro/Engineer 17}

A solid model of the part is created in the Part mode. Choose Interface from Part mode and Export from the Interface menu to create an igesout file. Pro/E creates a .prt file for all the parts created. This file lists the sequence of operations carried out in making the model in numerical form. This raw data is compiled into a 
relevant .inf (information) file. This inf file gives information like filename, units used (mm or inch), the features created, internal feature number, feature id, dimensions involved, the type of operations performed (Extrusion, Protrusion), etc. Pro/E also creates a. $\log$ file for all files that are imported and gives information about the IGES entities. It gives information about the IGES number associated with each entity (e.g. circular arc is represented by 100), entity name, the number of occurrences of that particular entity, whether all the entities present have been converted into IGES entity. It also gives the total number of curves, surfaces, trimmed surfaces it has identified as present in the model and total processing time. In order to accomplish the first step the exported file was imported back to Pro/E. The geometry and the plot looked the same. The .log files were checked for any error messages. It did not show any error messages, also the total number of entities remained the same. Thus we can see that the pre and post processors of Pro/E are consistent in translating the IGES files.

\section{Pro/E --.-> I-DEAS}

The model of the part created in Pro/E is exported to I-DEAS. To accomplish this we use the Part - Interface - Export menu options and then use the binary mode in file transfer protocol to transfer the igs file of the model to the server where IDEAS resides. To open the file in I-DEAS we create a new model file and use the File - Import - IGES (view independent) menu. The model is imported as a set of open surfaces, using the default tolerance available in I-DEAS (3.94E-4 inches or $0.01 \mathrm{~mm}$ ). Thus I-DEAS recreates the geometry based on the geometric definition, the topology and the required tolerance. If there are any errors during the transfer process, I-DEAS detects and lists the ill-defined or missing surfaces needed to be merged to form a solid. The $\log$ file lists all the errors, this. $\log$ file is stored as a .ipr (information processing report) file in the directory. In order to merge these open surfaces we open the file in the master surface module and use the autostitch menu to stitch all the individual surfaces and form a single solid. If this does not work we have to try doing the same using tighter tolerances so that the gaps in the open surfaces are reduced. This model imported from Pro/E as a set of surfaces is autostitched at a tolerance of $5.94 \mathrm{E}-4$, even though it was imported using a tolerance of 3.94E-4. The part now looks identical to the original part; its mass properties like volume and density are the same. Also the size of the igs file is same in both systems, ensuring completeness in the data transfer.

Although the size of the files and the overall geometry is same there is no one to one correspondence in the way the individual entities are identified and defined (as seen in the. $\log$ files of the individual systems, which gives the number, type and the IGES number of each entity) by the pre-processor (IGES translator in Pro/E) and the post-processor (IGES translator in I-DEAS). This means that some differences exist in the way the IGES translator, in each of the system interprets the individual entities that comprise the model, even though the overall geometry remains the same after the transfer. Thus the configuration of the IGES translator varies from system to system. 
(Pro/E --.->) I-DEAS ----> Pro/E

The third step in the procedure is carried out by exporting the I-DEAS solid (created in Pro/E) back to Pro/E. I-DEAS creates a .ipr file, which is checked for any error messages. The igs file is transferred to Pro/E by representing the IDEAS solid in the form of surfaces using the Surfaces Curves and Points option. This is because Pro/E prefers to import solids in the form of surfaces, as it has the capability to construct the solid from the imported data. Pro/E automatically creates a solid when sufficient data is available during import to create a solid, otherwise we can use Redefine - Fix Boundaries options or Merge quilts option to form a solid. Upon observation, this transferred model looks almost like the original with the mass properties, but the number of entities that make up the model (as seen in the new. $\log$ file) are more than that was originally transferred to I-DEAS. This is probably because of the autostitching performed on the model in I-DEAS.

Thus we see that for a part created in Pro/E we are able to move back and forth between Pro/E and I-DEAS using a standard exchange mechanism.

\section{Pro/E -..-> AutoCAD}

In order to check the compatibility between Pro/E and AutoCAD we have to follow the steps two and three of the test procedure as the first step has already been performed. First model the part in Pro/E, and then export it using the Part Interface - Export option. Here we have an option of either exporting it as a solid model or a drawing containing the different views generated from the model. If we want to export it as a drawing we need to use Drawing - Interface - Export option. There are several configuration options available that support the export of Pro/E parts and drawings to IGES. This helps in tailoring the IGES files to the receiving system's requirement. The iges_out_spl_crvs_as_126 configuration option sets all part geometry spline curves to IGESS entity $12 \overline{6}$ (B-SPLINE) when creating an IGES file. With these options available the igs file of the part is exported to AutoCAD (residing on a different server) using FTP. This file is viewed in AutoCAD using the Import option. As before we check the igs file of the part for any error messages or warnings during the data transfer. The .igs file gives only the numerical values of the entities that are transferred. The plan view of the solid model is seen in 2 dimensions. To view the 3D wireframe of the part we have to go to Views - Viewpoint and select the desired angle to view.

Thus AutoCAD does not have the capability to convert this into a solid or a surface model. So it is preferable to import the .igs file created from the drawing file containing the different views of the model.

\section{(Pro/E -..->) AutoCAD -.--> Pro/E}

The .igs file of the part imported into AutoCAD from Pro/E is created using the Export - Igs option. This file is transferred back to Pro/E using FTP. We observe that Pro/E is able to get back only the wireframe of the original solid model. We see from the. $\log$ file that the AutoCAD translator has defined the whole model in the form of wireframe entities like lines and circular arcs. Thus much of the 
information about the surfaces that was sent originally from Pro/E are lost in AutoCAD during translation. So AutoCAD is able to send only wireframe information back to Pro/E. Thus we see that the configuration and capabilities of the IGES translator of each of these systems are different. Pro/E has the capability to create a solid model of the part by using wireframe as the reference. For this we have to create datum planes for sketching and use the through option to construct the solid feature in the geometric tools menu. But this is a tedious process when bigger models are present.

Thus for a part created in Pro/E it is advisable to transfer 2D drawings into AutoCAD. It is not desirable to move the solid model back and forth between these two systems as much of the information on the surfaces and solids are lost in between due to the capabilities of the translators.

\section{I-DEAS 3.1}

A Solid model of the standard part is created in I-DEAS master series 3.1. For each model created two model files are generated by I-DEAS. This model file is exported using the File - Export - IGES option. I-DEAS has a variety of options for export depending on the receiving system called flavors. We can either use a standard flavor or a specific flavor depending on receiving system like AutoCAD, CADAM, CATIA, CAMAND, UNIGRAPHICS, VDA, INTERGR0APH, FordPDGS, GM-CGS. For the curves and surfaces the default flavor is B-spline, represented as Non Uniform Rational B-splines (NURBS). Solids can be exported as a set of surfaces, curves, points or model, space curves or model, space parameter space curves depending on the requirements of the receiving system. To perform the first step the model is exported using IGES and standard flavor as the option. Then this file is imported back to the system. In both cases the ipr file which gives the information processing report is checked for any errors.

There was no error and the geometry of the model was the same in both cases, indicating that the IGES processor of I-DEAS is able to transfer CAD data completely without change of content and functionality.

\section{I-DEAS --.-> Pro/E}

The standard part is created in I-DEAS using the Master Modeler and Design menus. This part is exported using the File - Export - IGES option. Since I-DEAS does not have a specific flavor for Pro/E as such we use the IGES option. Here we use the option of representing the I-DEAS solid as a set of surfaces, curves and points in IGES. Use the default tolerance of 3.94E-4. The log file is checked for errors or warning messages. There were no errors recorded by the pre-processor in reading the IGES entities. This file is run in Pro/E, and again the $\log$ file is checked for any errors. There were no error messages. The plots look the same, the geometry is correct.

Thus indicating that the post-processor (Pro/E translator) is able to read the IGES entities sent by the pre-processor (I-DEAS translator). 
(1-DEAS ---->) Pro/E -.--> I-DEAS

The above file in Pro/E (Originally modeled in I-DEAS), has to be sent back to IDEAS. The Interface - Export option is used in Pro/E to create the igesout file for the part. There were no errors recorded. This is then sent back to I-DEAS using FTP. The size of the igs file is the same in both the systems, thus indicating that the completeness of the data transfer. The part, which is transferred as a set of surfaces, has to be stitched using the Master surface module to form a solid. The resulting solid is compared to the original solid that was modeled. It has the same geometry, mass properties are also the same. This shows that there was no loss of information during the transfer and also that the translator is able to identify the entities created by the IGES processor of Pro/E. Thus a model created in I-DEAS can be sent back and forth to Pro/E effectively.

\section{I-DEAS --.-> AutoCAD}

The solid model created in I-DEAS is exported to AutoCAD (in a different server) using the AutoCAD flavor option. The $\log$ files are checked for any errors. The file is then read in AutoCAD using the Import - igsin menu. The 2D view of the model appears in AutoCAD. The 3D wireframe of the solid created in I-DEAS is viewed in AutoCAD using the Views - Viewpoint menus. If the AutoCAD flavor option in I-DEAS is not used we see a distorted image of the geometry when transferred to AutoCAD. This is because the AutoCAD is not able to recognize the surface information sent by I-DEAS processor. This is evident upon checking the mass properties (which says that the part volume is zero). I-DEAS also has the capability to convert the solid model created in the Master modular to 2D drawings. To do this we have to open the same model in the Drafting Setup module then use the Create Layout icon to enter the information to create the drawing of the parts already modeled, then use the Move icon to reposition any dimensions or make sections, and add annotations to the drawing and go to the Drafting Detailing module. From here the drawing can be exported to AutoCAD using the AutoCAD flavor. These drawings can be viewed in AutoCAD without any changes.

\section{(I-DEAS' -...) AutoCAD -... I-DEAS}

Using the export menu in AutoCAD the part is transferred back (using FTP) to IDEAS. We get the wireframe of the original model, but the $\log$ file shows that the AutoCAD processor has interpreted the entire model as a collection of only two entity types namely lines and circular arcs. There are many open edges in the part that cannot be modified because I-DEAS does not have enough information to generate a solid model from this IGES file from AutoCAD. Although I-DEAS transferred information about the curves (B-splines), and surfaces to the AutoCAD processor, much of the information about the model is lost when a igs file (of the same part) created in AutoCAD is transferred back to I-DEAS.

Thus indicating that the IGES translator of AutoCAD has the capability to transfer only 2D and 3D wireframe information but not about surfaces and solids. 


\section{AutoCAD}

The standard part is drawn in AutoCAD. This file is exported using File - Export Igesout option. AutoCAD 13 supports IGES 4.0 and it requires the files to be exchanged in fixed length ASCII format. The AutoCAD translator (Version 3.04) creates the igs file. This is then imported back to AutoCAD using the File - Import - Igesin option. The drawings appear exactly the same, the size of the igs file is the same, and there are no changes in the text and annotations.

Thus Igesin and Igesout options of AutoCAD support the translation of drawings and preserve the geometry and functionality of the entities.

\section{AutoCAD -..> Pro/E}

The drawing created in AutoCAD is exported to Pro/E, the drawing is regenerated by the translator of Pro/E, with all the text, dimensions and annotations, without any errors. Pro/E also regenerates the 3D wireframe created in AutoCAD.

\section{(AutoCAD …>) Pro/E _...> AutoCAD}

The above part in Pro/E (from AutoCAD) is exported using the Interface - Export option and transferred to AutoCAD using FTP (ASCII mode). The file is viewed in AutoCAD using the Igesin. All dimensions and annotations are visible, but AutoCAD is not able to define the drawing extents. Thus the wireframe originally created in AutoCAD, when transferred back from Pro/E is regenerated without any errors. We see that the translators of AutoCAD and Pro/E are able to recognize and interpret the IGES files created by one another correctly.

\section{AutoCAD -..-> I-DEAS}

The part created in AutoCAD is exported to I-DEAS. We first transfer the drawing and then the wireframe geometry of the part to I-DEAS. Text and annotations are lost when we transfer drawings.

\section{(AutoCAD --.->) I-DEAS --->> AutoCAD}

The file is transferred back to AutoCAD, from I-DEAS. Here again there are no annotations and text. But the overall file transfer is smooth and we get whatever information that was sent originally from AutoCAD back.

(ii) STEP

The same testing procedure is used to test the interoperability of Pro/E and IDEAS, by using the Standard For Exchange Of Product Model Data (STEP) standard as the data exchange mechanism. Both Pro/E and I-DEAS support the AP 203IS of the ISO 10303 STEP format. This format provides file exchange for Configuration Controlled three-dimensional designs of mechanical parts and assemblies with advanced B-Rep solids, Non-Topological surface and Wireframe.

\section{Pro/Engineer 17}

The solid model of the part is created in part mode and then exported to STEP format using Interface - Export option. This procedure is same as IGES except 
that we select the STEP menu from the Export option. Here we have the choice of exporting the part as a wireframe, surfaces, solid, shells, and datum curves. We select the solid option. Pro/E creates a .stp file and a $\log$ file, which contains the list of STEP entity types used in creating the exported file.

To carry out the Step One of the testing procedure this .stp file is imported back to Pro/E. This is done by choosing Interface - Import option and then select STEP menu. Then create a Co-ordinate system to locate the STEP data. The system starts processing the STEP data. On checking the $\log$ file the number of entity types that make up the part remains the same, the solid model and its properties like mass, density, etc. remain the same. Thus we see that the STEP translator is consistent in parsing the input data created by itself.

\section{Pro/E ----> I-DEAS}

The Step Two of the testing procedure is then carried out by transferring the exported STEP file (.stp) to I-DEAS using the file transfer protocol. The exported solid model from Pro/E is imported by I-DEAS using the geometry translators menu. We observe that the solid model is transferred in its entirety with the mass properties remaining the same. Thus the STEP translator of I-DEAS is able to transfer the $\mathrm{CAD}$ data without losing much information.

\section{(Pro/E ---->) I-DEAS $\rightarrow->>$ Pro/E}

This follows the Step Three of the testing procedure, wherein the part created in $\mathrm{Pro} / \mathrm{E}$ is transferred back to Pro/E from I-DEAS. Here we use the Interface Import option to import the STEP data from I-DEAS after selecting the Coordinate system. Pro/E gives out an error message saying "could not construct feature geometry" as the translator is not able to process some entities that help in finalizing the topology.

\section{I-DEAS}

The solid model of the standard part is created first and then the Step One of the testing procedures is performed. For this we switch to geometry translators, open the model file that contains the part then use the Export option. Complete translation is possible using the default option for export. When we export the part a relationship is created between the exported part and the resulting STEP file. All user attributes will be mapped to the Configuration management data which gives information on the part, part number, name descriptor, etc. The configuration management data keeps pertinent information about the part even as the part moves from different suppliers and vendors. When we export two files are created namely the .step and .opr (output processing report) files. The .opr file gives the list of parameters that are set and the list of STEP entities.

This file is imported back by I-DEAS. For this we switch back to geometry translators, open the model file that contains the part and use the Import option. It should be noted that only single parts can be imported into I-DEAS from STEP translator. The system automatically creates a ipr (input processing report) file. We see that the model remains the same in form and has the same mass properties. 
Thus indicating that the STEP translator is consistent in translating data created by itself.

\section{I-DEAS ----> Pro/E}

The STEP file that was exported by I-DEAS is imported by Pro/E after creating the co-ordinate system. We observe that the part is translated without any change in the shape, mass properties, etc. Thus indicating that the STEP translators of Pro/E and I-DEAS are able to communicate effectively. By this we have completed the Step Two of the testing procedure successfully.

\section{(I-DEAS --->) Pro/E ---> I-DEAS}

This is the Step Three of the testing procedure. The STEP file of the part from IDEAS is created in Pro/E using the Export option. This is then transferred back to I-DEAS using the geometry translators and import option. I-DEAS gives out a message saying that the system has encountered errors parsing the input file. It also says that mapping of a particular entity to and from STEP is not supported by the STEP translator.

Thus we observe that both Pro/E and I-DEAS are able to transfer the STEP file to itself (Step One of testing procedure) and to the other system (Step Two of testing procedure) successfully. But is not able to translate the data back to the native system from another system.

\section{CONCLUSION}

We see that Pro/E is compatible with both I-DEAS and AutoCAD, with regard to back and forth transfer of CAD data using IGES. But AutoCAD is not efficient in transferring data on surfaces and solids. This could be due to the inherent differences in the capabilities of the individual CAD software or due to the way the part is modeled as there are no modeling standards available. STEP translators are efficient in one way transfer of data between I-DEAS and Pro/E, but are not able to aid in back and forth transfer of CAD data between these software. Thus there exists some differences in the interpretations of the STEP standard by different CAD/CAM software vendors.

\section{REFRENCES}

1. Elwood, G. P. (1993) Interoperability acceptance testing methodology version 1.01

2. AutoDesk Inc. (1996) AutoCAD Release 13 guide, AutoDesk Inc. Publications, California.

3. Lawry, M.H (1995) I-DEAS Master series guide, OnWard Press, New Mexico.

4. Parametric Technology Corporation. (1996) Pro/Engineer Fundamentals, Massachusetts 
5. National Aerospace Standard, (1969), " Uniform cutting tests-NAS series metal cutting equipment specification," Aerospace association of America Inc., Washington D.C. 